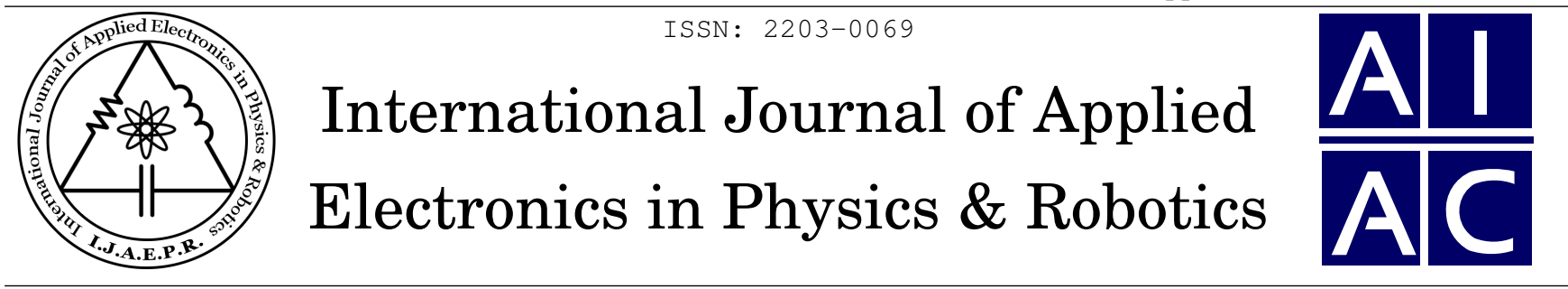

Research Article

\title{
Dynamic Analysis of GaAs-based Quantum Cascade Laser
}

\author{
Vahed Servati $^{1}$, Kambiz Abedi ${ }^{2}$, Mohammad Ali Shariatzadeh ${ }^{3}$, Vahid Tabatabaie ${ }^{3, *}$
}

(1) Department of Electrical Engineering, Ahar Branch, Islamic Azad University, Ahar, Iran.

(2) Department of Electrical Engineering, University of Shahid Beheshti, Tehran, Iran.

(3) Department of Nanophotonics Engineering, Tabriz University (Pardis Aras), Tabriz, Iran.

\section{Article history:}

Received 25 August 2013

Reviewed 24 September 2013

Revised 08 October 2013

Accepted 10 October 2013

Published 11 October 2013

\begin{abstract}
The quantum cascade lasers are such unipolarity lasers that have the emissivity from mid infrared to far infrared range. In this paper the dynamic effects of quantum cascade lasers (QCL) GaAs /AlGaAs characteristics in the mid-infrared range of $\lambda=9 \mu \mathrm{m}$ is investigated by solving the numerical equations. Calculating of the spontaneous emission rate changes, its effects on the number of carriers, the number of photons, and the output power are the objectives of the presented study. Finally, the output power changes, by increasing the number of stages in the quantum cascade laser, have been studied. The obtained results showed that, the increase of spontaneous emission rate parameter causes delay in the laser output. In addition, there is a direct relationship between the number of stages and the output power.
\end{abstract}

Keywords: quantum cascade lasers, spontaneous emission, GaAs, numerical equations, laser rate equation.

\section{Introduction}

Quantum cascade lasers (QCL) are such semiconductor lasers that are made of thin films with heterogeneous structures where the thickness of these layers and the barrier height determine the output wavelength ${ }^{[1]}$. One of the important differences between the operation of these lasers and semiconductor ones is that in the quantum cascade lasers, only one carrier is used ${ }^{[2,3]}$. In other words, the quantum cascade lasers are such unipolarity lasers that have the emissivity from mid infrared to far infrared range which radiative transitions between discrete levels into the conduction band is done ${ }^{[2]}$. Thus, without needing to change the material used, only by adjusting the layers thickness, the wide range of wavelengths can be received from the system ${ }^{[4]}$. In addition, the cascades of these lasers are periodic. In fact, to produce high gains, the series the modules is essential. By series the modules, electrons can be allowed to move from one period to another period which caused to participate in producing the output power.

There are several early studies about the operation of the quantum cascade laser emitted light, which one of the oldest presented by Faist et al. in 1994 that designed and demonstrated a semiconductor injection quantum cascade laser which was different from diode ones ${ }^{[3]}$. Nowadays, the quantum cascade lasers are made by using triple material combinations.

GaInAs/AlInAs/InP is considered as a base compound

* Corresponding author: V. Tabatabaie

๘: +98 (0)9149162932

凶: vahid.tabatabaie@yahoo.com which for the mid-infrared lasers shows its best performance. The compound of $\mathrm{GaAs} / \mathrm{AlGaAs} / \mathrm{GaAs}$ is used for terahertz lasers and the structures $\mathrm{AlSb} / \mathrm{InAs} / \mathrm{GaSb}$ and $\mathrm{AlSb} / \mathrm{InAs}$ which are suitable for microwave applications.

In this research, the dynamic effects of quantum cascade lasers GaAs/AlGaAs characteristics in the midinfrared range of $\lambda=9 \mu \mathrm{m}$ is investigated by solving the numerical equations. Then, we have studied the spontaneous emission rate changes, its effects on the number of carriers, the number of photons, and the output power. Finally, the simulation results showed that the increase of spontaneous emission rate parameter causes delay in the laser output. In addition, there is a direct relationship between the number of stages and the output power.

\section{Rate Equations Model}

Developing of the rate equation model for a coupled electromagnetic and electronic system can obtain some important structural parameters ${ }^{[5]}$. The rate equations for the number of electrons in the third layer $N_{3}$, the second layer $N_{2}$, and the number of photons $N_{p h}$ in the cavity is obtained as:

$$
\frac{d N_{3}}{d t}=\eta W L \frac{J}{e}-\frac{N_{3}}{\tau_{3}}-\Gamma \frac{c}{\mathrm{c} \sigma_{32}}\left(N_{3}-N_{2}\right) N_{p h}
$$

$$
\begin{aligned}
\frac{d N_{2}}{d t} & =(1-\eta) W L \frac{J}{e}+\frac{N_{3}}{\tau_{32}}-\frac{N_{2}}{\tau_{21}} \\
& +\Gamma \frac{\dot{c} \sigma_{32}}{V}\left(N_{3}-N_{2}\right) N_{p h}
\end{aligned}
$$




$$
\frac{d N_{p h}}{d t}=N \Gamma \frac{\dot{c} \sigma_{32}}{V}\left(N_{3}-N_{2}\right) N_{p h}-\frac{N_{p h}}{\tau_{p}}+\beta \frac{N_{3}}{\tau_{s p}}
$$

Where $\eta$ denotes the diffusion coefficient to higher levels of laser, $e$ is the electronic charge, $J$ represents the electrons current density in the third layer, $W$ and $L$ are the width and length of the cavity respectively; $V$ is equal to $N W L L_{p}$ which is the volume of the cavity, where $L_{p}$ and $N$ are the length of a single stage of laser structure and the number of gain stages, respectively; $c=\frac{c}{n_{\text {eff }}}$ is the velocity of light in medium, where $n_{\text {eff }}$ is the effective refractive index of cavity and equals 3.27 , while $\eta_{\text {eff }}$ denotes the effective diffusion coefficient to higher levels of laser that equals 0.3 (see Table 1 ); $c$ represents the light speed in vacuum; $\beta$ denotes the spontaneous emission factor; $\Gamma$ and $\sigma_{32}$ are the mode confinement and the spontaneous emission factor, respectively.

$$
\sigma_{32}(T)=\frac{4 \pi e^{2} z_{32}^{2}}{\eta_{\mathrm{eff}} \varepsilon_{o} \lambda\left(2 \gamma_{32}\right)}
$$

In (4), $e z_{32}$ denotes the dipole matrix element; $\lambda$ is the emission wavelength, $\varepsilon_{o}$ represents the permittivity of vacuum coefficient, and $2 \gamma_{32}$ is the full width at the half maximum. The photon lifetime tp in the cavity equals $\tau_{p}^{-1}=\hat{c}\left(\alpha_{w}+\alpha_{m}\right)$, where $a_{w}$ denotes the waveguide losses in the cavity, and $a_{m}$ is the losses mirror.

\section{Conduction Band Structure}

In Fig. 1, for generating 33 terahertz frequency, the conduction band structure of the GaAs/AlGaAs to the wavelength $\lambda=9.1 \mu \mathrm{m}$ is plotted where GaAs represents its active region and $\mathrm{AlGaAs}$ denotes the barrier region. The arrangement of layers in the nanometer-scale as follows, started from the barrier layer: 4.6/ 1.9/ 1.1/ 5.4/ 1.1/ 4.8/ 2.8/3.4/ 1.7/3/ 1.8/2.8/ 2/3/ 2.6/3; where the bold numbers represent the barriers and the others denote the holes.

In Fig. 2, the effect of the spontaneous emission on the carriers and photons density are shown, where the spontaneous emission changes caused delays in the output carriers photons generation ${ }^{[6]}$. Unlike typical lasers which the number of photons is less than the number of carriers, in the quantum cascade lasers due to the cascade, more photons are produced ${ }^{[7]}$.

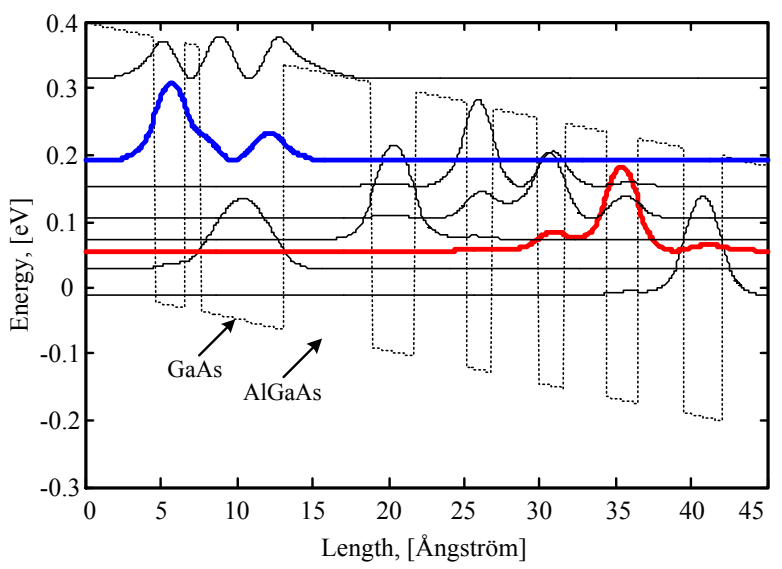

Fig. 1. The conduction band structure of GaAs/AlGaAs (a)

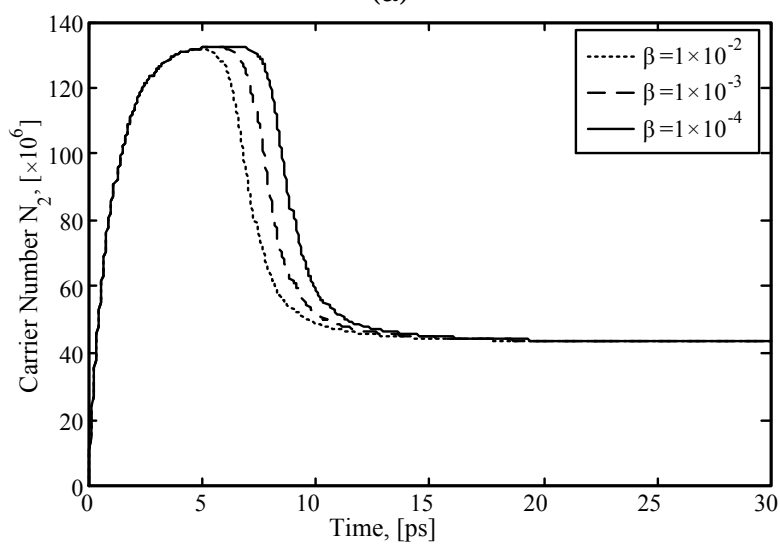

(b)

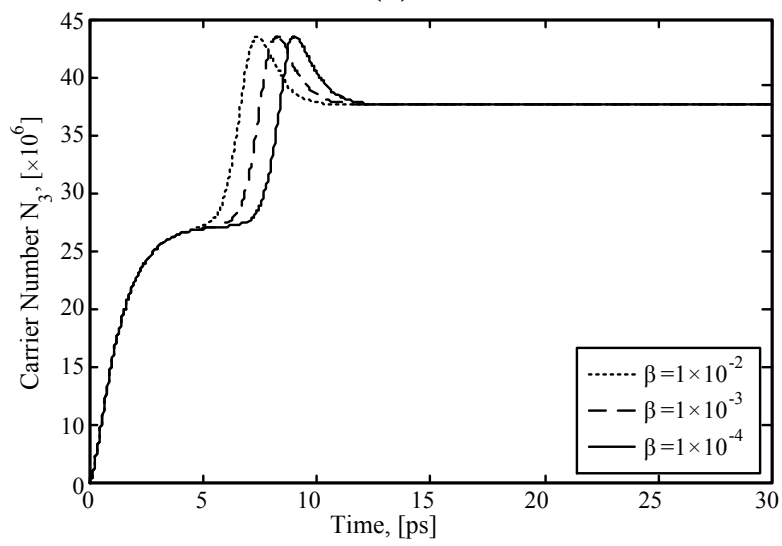

(c)

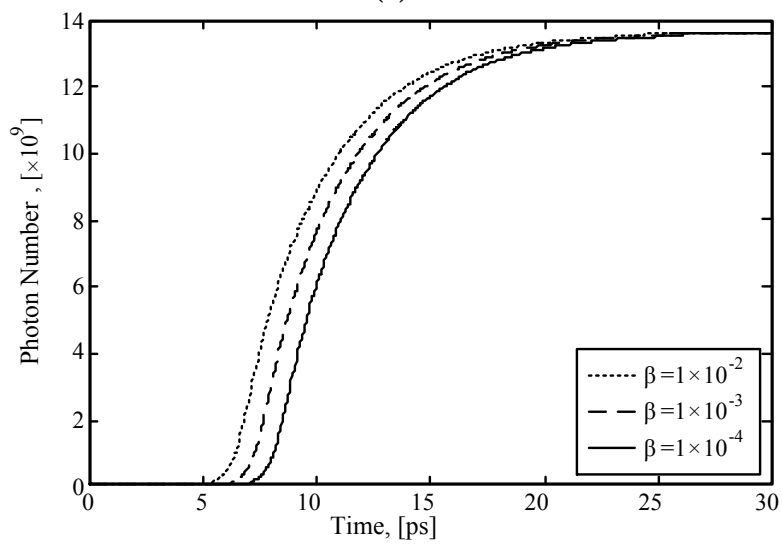

Fig. 2. The spontaneous emission on the output (a) carriers' density in the second layer, (b) carriers' density in the third layer, and (c) photons' density

\section{Output Power}

Power equation (5), the relationship between the output power and the number of photons, is explaining the amplification of the QCL light which is obtained as follows ${ }^{[6]}$.

$$
P_{\text {out }}(T)=\frac{\eta_{o} \hbar \omega N_{p h}}{\tau_{p}}
$$

In the above equation, $\hbar \omega$ is the emitted photon energy, and $\eta_{o}$ is the output power coupling coefficient which can be obtained from $(6)^{[5]}$.

$$
\eta_{o}=\frac{\left(1-R_{1}\right) \sqrt{R_{2}}}{\left(1-R_{1}\right) \sqrt{R_{2}}+\left(1-R_{2}\right) \sqrt{R_{1}}} \times \frac{\alpha_{m}}{\alpha_{w}+\alpha_{m}}
$$


Table 1. The values used in the simulations.

\begin{tabular}{ll}
\hline Parameters & Values \\
\hline$\eta$ & 0.91 \\
$\tau_{p}$ & $3.3 \mathrm{ps}$ \\
$V=N W L L_{p}$ & $50 \times\left(34 \times 10^{-6}\right) \times\left(1 \times 10^{-3}\right) \times\left(45 \times 10^{-9}\right)$ \\
$z_{32}$ & $1.7 \mathrm{~nm}$ \\
$\tau_{s p}$ & $36 \mathrm{~ns}$ \\
$\Gamma$ & 0.3 \\
$n_{\text {eff }}$ & 3.27 \\
$\eta_{\text {eff }}$ & 0.3 \\
$\alpha_{w}$ & $20 \mathrm{~cm}^{-1}$ \\
$\tau_{3}$ & $1.3 \mathrm{ps}$ \\
$\tau_{32}$ & $2.1 \mathrm{ps}$ \\
$\tau_{21}$ & $0.3 \mathrm{ps}$ \\
$\gamma_{32}$ & $6 \mathrm{meV}$ \\
\hline
\end{tabular}

where $R_{1}$ and $R_{2}$ represent the reflecting powers of facets 1 and 2 , respectively. The dependence of the output power to the spontaneous emission rate and the number of stages can be obtained by solving these related equations.

\section{Numerical Results and Discussion}

The simulation results show that by increasing the spontaneous emission rate cause reducing the time delay and laser threshold current is also reduced. However, because of the dependence of these effects to the temperature and the uncontrollable of these effects, these effects are known as undesired factors in laser. The values used in the simulations are derived from references ${ }^{[5,8-10]}$ and presented in Table 1.

Fig. 3(a) shows the dependence of the output power to the spontaneous emission rate. According the Fig. 3(a), there is a reverse relationship between the rate of spontaneous emission and the threshold current. In other words, increasing of the spontaneous emission rate causes the threshold current reduction. In Fig. 3(b), the increase of the number of stages on the output power based on the current injection changes with $\eta=1$ is shown.

The quantum cascade laser output power varies approximately linearly by the current injection. It is observed that increasing the number of stages causes the increase of the output power; thereby the power slope can increase by increases of the number of stages.

\section{Conclusion}

In summary, in the presented model the effects of spontaneous emission and increasing of the number of stages on the quantum cascade laser performance in the midinfrared is shown. In this model, the assumptions are the dependence on photons and carrier densities, and the output power. Simulation results show that the increase of spontaneous emission rate parameter causes delay in the laser output. Meanwhile, increase of the number of stages causes the output power be increased.

\section{REFERENCES}

[1] B. S. Williams, S. Kumar, Q. Hu, and J. L. Reno, "Operation of terahertz quantum-cascade lasers at $164 \mathrm{k}$ in pulsed mode and at $117 \mathrm{k}$ in continuous-wave mode," Opt. Express, vol. 13, no. 9, pp. 3331-3339, 2005.
[2] A. Hamadou, "Optical external efficiency calculation for mid-infrared quantum cascade laser," Optica Applicata, vol. 41, no. 3, pp. 717-725, 2011.

[3] J. Faist, F. Capasso, D. L. Sivco, C. Sirtori, A. L. Hutchinson, and A. Y. Cho, "Quantum cascade laser," Science, vol. 264, no. 5158, pp. 553-556, 1994.

[4] A. Bacou, A. Hayat, V. Iakovlev, A. Syrbu, A. Rissons, J.-C. Mollier, and E. Kapon, "Electrical modeling of longwavelength vcsels for intrinsic parameters extraction," Quantum Electronics, IEEE Journal of, vol. 46, no. 3, pp. 313-322, 2010.

[5] B. Mirzaei, A. Rostami, and H. Baghban, "Terahertz dualwavelength quantum cascade laser based on $\mathrm{GaN}$ active region," Optics \& Laser Technology, vol. 44, no. 2, pp. 378383, 2012.

[6] X. Gao, D. Botez, and I. Knezevic, "Phonon confinement and electron transport in gaas-based quantum cascade structures," Journal of Applied Physics, vol. 103, no. 7, pp. $073101-073$ 101, 2008.

[7] R. Paiella, Intersubband transitions in quantum structures. McGraw Hill Professional, 2006.

[8] A. Hamadou, J.-L. Thobel, and S. Lamari, "Modelling of temperature effects on the characteristics of mid-infrared quantum cascade lasers," Optics Communications, vol. 281, no. 21 , pp. 5385-5388, 2008.

[9] A. Hamadou, S. Lamari, and J.-L. Thobel, "Dynamic modeling of a midinfrared quantum cascade laser," Journal of Applied Physics, vol. 105, no. 9, pp. 093 116-093 116, 2009.

[10] G. Strasser, S. Gianordoli, L. Hvozdara, W. Schrenk, and E. Gornik, "Intersubband and interminiband GaAs/AlGaAs quantum cascade lasers," Physica E: Low-dimensional Systems and Nanostructures, vol. 7, no. 1, pp. 1-7, 2000.

(a)

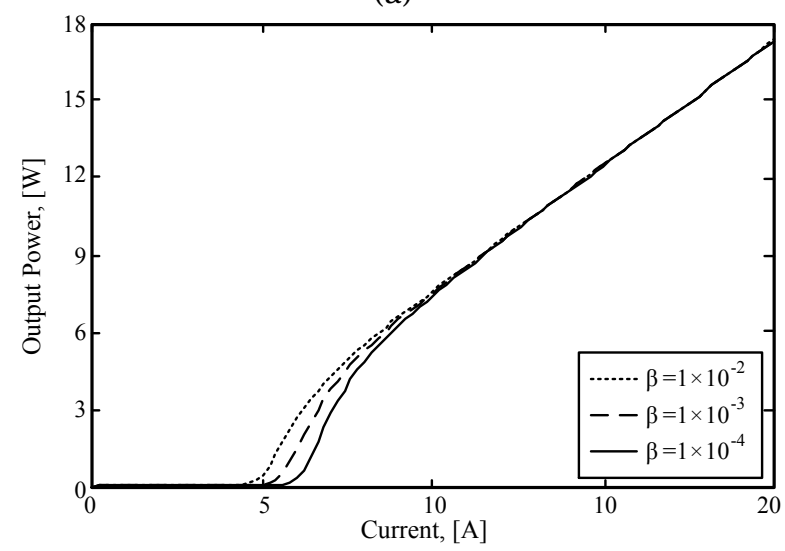

(b)

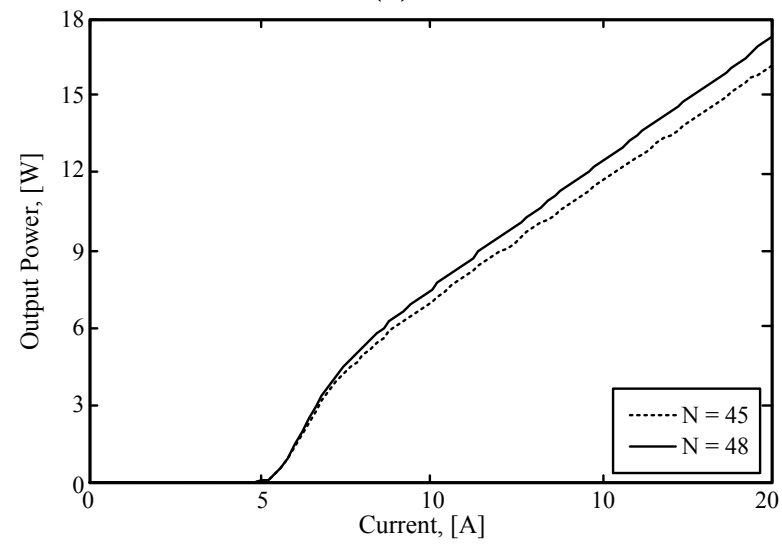

Fig. 3. (a) The spontaneous emission and (b) the effect of increasing of the stages numbers on the output power 